\title{
CD-ROM REVIEWS
}

\section{CABI Publishings Topics in International Health: Sickle Cell Disease}

ickle cell disease, one of the most common genetic disorders in the world, af$\checkmark$ fects millions of people worldwide and 70,000 to 80,000 people in the United States. It is particularly common in people of ancestry from sub-Saharan Africa and the Middle East. Sickle cell disease affects almost every major organ and, in addition, is a prototype for advances in our understanding of genetic medicine and gene regulation.

Because of sickle cell disease's importance from a public health perspective, it is only appropriate that the Welcome Trust, as part of the series of Topics in International Health, has developed a new interactive CDROM. Three physicians, from Jamaica, England, and Nigeria, with diverse clinical experience, have authored 16 interactive tutorials and 275 images summarizing various aspects of the pathophysiology and clinical features of sickle cell.

The CD-ROM begins with an overview of sickle cell disease, which is followed by a discussion on hemoglobin structure and synthesis, the interaction of hemoglobin $\mathrm{S}$ with hemoglobin $\mathrm{C}$ and the Thalassemias, and genetics, hematological findings, diagnostic tests and common complications of Sickle Cell disease. There are specific chapters on lung, spleen, renal and eye pathophysiology. There is specific information about sickle cell disease's impact on malaria, and common infections in patients with sickle cell disease.

This CD-ROM provides an enjoyable and interactive teaching tool that would be difficult to duplicate in a traditional text. On almost every page of the 16 tutorials there is an image or video with patient pictures, hematological slides, or schematic demonstrations. The images are well labeled with descriptions. The text is organized into outline format and clicking underlined text or a button under each topic can retrieve additional information. Each page is also equipped with several buttons on the bottom for easy access to different topics. For instance, a "content" button will redirect you to the table of contents page and allow you to skip to another page. A notepad is provided for taking notes and a glossary is built in that can be accessed from every page in the tutorial. References are also included for additional readings, although abstracts of these articles are not included. At the end of each section there is an assessment quiz. Correct answers and explanations automatically appear once an answer is made. There is a summary of the chapter at the end of each tutorial.

This CD-ROM primarily covers the pathophysiology, at the organ level, of sickle cell disease. It is unfortunate that with the power of this CD-ROM, it is not more encompassing. There is little information on the basic science of sickle cell (e.g. mechanisms of hemoglobin switching, ion transport in the sickle red cell, the role of nitric oxide and leukocyte adhesion in the pathology of sickle cell disease). There is unfortunately also little information on very clinically relevant topics, such as treatment with hydroxyurea, or pain management. It is, however, a tribute to the excellence of the presentation of this CD-ROM that one misses the opportunity of reading about these topics. Because of its outline format, this CD-ROM is an ideal review or teaching aid for medical students and public health professionals. However, for those seeking detailed and advanced knowledge on the pathophysiology and management of the disease, additional reading is required.

\author{
Qing Li, M.D., Ph.D \\ Lawrence B. Gardner, M.D. \\ Division of Hematology \\ Department of Medicine \\ Johns Hopkins University
}

\section{CABI Publishings Topics in International Health: Tuberculosis}

$\mathrm{D}$ espite curative anti-tuberculous chemotherapy since the mid $20^{\text {th }}$ century, rates of tuberculosis continue to rise in many parts of the world. Human immunodeficiency virus co-infection has complicated the tuberculosis epidemic with globally increasing case rates despite directly observed therapy. Emergence of drugresistant strains has reinforced the need for better strategies in tuberculosis prevention, control, and treatment. This CD-ROM provides an excellent overview of tuberculosis with information divided into nine sections: etiology and transmission, immunology, pathogenesis, epidemiology, prevention and control, clinical features, role in the HIVinfected, diagnostic tests, and treatment.

The information is presented in three different formats to allow for quick reference as well as prolonged study: ten active tutorials, a glossary of common terms, and a beautiful collection of 619 images that can be downloaded. The interactive format of the tutorials keeps the readers attention with animated graphics and frequent fact assessments. At the end of each tutorial, summaries are pre- sented that reiterate main points and allow for a more cursory overview of the material. The transmission dynamics of Mycobacterium tuberculosis and the importance of cellmediated immunity in the control of the disease are highlighted. The consequences of impaired cellular immunity in the HIVinfected host are contrasted with immune mechanisms in the normal host. The authors take advantage of this educational medium by presenting typical features of disease, diagnosis, treatment, and prevention with more photographs and graphics than would be found in a textbook. Overall, the material stresses the growing stature of this organism in public health.

The major drawbacks to this CD-ROM are those inherent in this type of presentation format. The level of detail varies from section to section. In addition, despite a large collection of photographs and slides, some of the text and photographs are repeated in multiple sections. The outline format although bulleted and clearly graphically presented is occasionally more disjointed and difficult to understand than textbook prose.

This CD-ROM will have wide appeal among medical providers and public health students with a specific interest in tuberculosis, and basic scientists interested in the clinical aspects of the disease. Although the basic immunological mechanisms of disease patho- genesis are covered, the molecular virulence mechanisms of the tubercle bacillus are not discussed. In addition, molecular techniques such as DNA fingerprinting, which track the epidemiology of tuberculosis are not described. Although the microbiology of $\mathrm{Myco}$ bacterium tuberculosis is briefly presented, specific drug resistance patterns and mechanisms are likewise omitted. Introductions to these topics would have provided an even larger survey of the scope of tuberculosis research and will hopefully be provided in any subsequent updates.

This CD-ROM provides an excellent overview of many aspects of Mycobacterium tuberculosis pathogenesis and clinical disease. Based on the current epidemiological trends, the need for greater awareness of the impact, scope and nature of this important infectious disease remains a priority. The interactive format and pictorial presentation allow for new and unique methods of learning for those interested in a review of tuberculosis.

\footnotetext{
Yukari C. Manabe, MD Assistant Professor, Department of Medicine Division of Infectious Diseases Johns Hopkins University 424 North Bond Street, Rm108 Baltimore, $M D$

ymanabe@jhmi.edu
} 